\title{
LA VIDEOGRABACIÓN COMO DETONANTE DE REFLEXIÓN EN LOS EDUCADORES
}

\author{
Esteban García Hernández* \\ Universidad Pedagógica Nacional del Estado de Chihuahua
}

\section{RESUMEN}

Este artículo expone la etapa inicial y los primeros resultados de la investigación en torno a procesos de reflexión sobre la práctica docente que realizan profesores de grupos de niñas, niños y adolescentes de familias jornaleras migrantes en Chihuahua. Conocer esas reflexiones es el propósito de la indagación, donde las videograbaciones de sus clases han funcionado como desencadenantes del diálogo y la reflexión. El trabajo se efectuó desde un enfoque cualitativo durante el ciclo escolar agrícola 2017 en centros escolares chihuahuenses. Los resultados iniciales muestran que los relatos docentes constituyen reflexiones descriptivas, que establecen razones o justificaciones basadas en opiniones o experiencias directas y propias.

Palabras Clave: formación de educadores, educación básica, reflexión, niñez migrante.

\section{VIDEO RECORDING AS A TRIGGER FOR \\ REFLECTION IN EDUCATORS}

\section{Abstract}

This article exposes the initial stage and the first results of the investigation around processes of reflection on the teaching practice carried out by teachers of groups of girls, boys and adolescents of migrant day laborer families in Chihuahua. The purpose of the inquiry is knowing these reflections, and the video recordings of the classes of these teachers have worked as triggers for dialogue and reflection. The work was carried out from a qualitative approach during the agricultural school year 2017 in schools in Chihuahua. The initial results show that the teachers' stories constitute descriptive reflections, which establish reasons or justifications based on opinions or direct and own experiences.

KEYWORDS: educator training, basic education, reflection, migrant children.

DOI: https://doi.org/10.25145/j.qurricul.2019.32.06

Revista Qurriculum, 32; junio 2019, pp. 99-113; ISSN: e-2530-8386 


\section{INTRODUCCIÓN}

Este artículo es un producto preliminar del trabajo de indagación que se realiza en el Doctorado en Desarrollo Educativo con énfasis en formación de profesores, capítulo noroeste, de la Universidad Pedagógica Nacional del Estado de Chihuahua (UPNECH), en México. La investigación se ubica en la Línea de Generación y Aplicación del Conocimiento (LGAC) de Desarrollo Profesional. La temática a tratar se refiere a la formación de educadores que atienden a niñas, niños y adolescentes (NNA) de familias jornaleras agrícolas migrantes en el estado de Chihuahua, México.

Su elaboración se basó en algunos resultados obtenidos en una primera etapa de la investigación. Durante el ciclo agrícola 2017, se efectuó un acercamiento a 11 centros escolares de educación básica que atienden población migrante en siete municipios chihuahuenses, en el norte de México, ver tabla 1.

\begin{tabular}{cccc}
\hline \multicolumn{2}{c}{$\begin{array}{c}\text { TABLA 1. MUNICIPIOS, NÚMERO DE CENTROS ESCOLARES Y GRUPOS } \\
\text { EN LOS QUE SE REALIZARON ENTREVISTAS }\end{array}$} \\
\hline Municipios & CENTROS ESCOLARES & GRUPos \\
\hline Ascensión & 1 & 9 \\
\hline Cuauhtémoc & 3 & 6 \\
\hline & Delicias & 1 & 1 \\
\hline & Guerrero & 2 & 3 \\
\hline & Meoqui & 2 & 3 \\
\hline & Ojinaga & 1 & 2 \\
\hline & Rosales & 1 & 1 \\
\hline Total & & 11 & 25 \\
\hline
\end{tabular}

Fuente: elaboración propia.

De acuerdo con la base de datos del Sistema Nacional de Control Escolar para Migrantes (SINACEM), consultada en septiembre del 2017, en el estado de Chihuahua se encontraban inscritos 1298 NNA de familias migrantes (641 hombres y 657 mujeres), en 30 escuelas pertenecientes al Programa para la Inclusión y la Equidad Educativa (PIEE), distribuidas en 17 municipios de la entidad. En 13 centros escolares se contaba con alumnos inscritos en los tres niveles de educación básica; en los restantes, únicamente en preescolar y primaria. Se muestran aquí los resultados en torno al siguiente par de objetivos específicos, planteados para esta etapa inicial:

*E-mail: estebangarcia_h@yahoo.com. Es estudiante de doctorado de la Universidad Pedagógica Nacional del Estado de Chihuahua. Sus intereses profesionales se orientan hacia la investigación educativa de la formación docente y continua de la educación básica rural, particularmente en la educacion de niñas y niños jornaleros agrícolas migrantes. 
- Definir el perfil profesional de un grupo de educadores de centros escolares para migrantes del estado de Chihuahua.

- Identificar las características que los educadores de migrantes atribuyen a su práctica pedagógica.

Por principio metodológico, la investigación parte de las percepciones que han expresado los educadores de población migrante, para comprender, desde sus voces y experiencias, cómo es la educación que se ofrece a la niñez migrante, e identificar con ellos sus necesidades de formación profesional.

\section{SitUACIÓN GENERAL DE LA EDUCACIÓN DIRIGIDA A LA NIÑEZ MigRANTE}

El artículo $3 .^{\circ}$ de la Constitución Mexicana establece el derecho de todos los individuos a recibir una educación de calidad. Con apego a esta ley, el Estado debe asegurar a la población de niños y jóvenes acceso universal a la escuela, en condiciones que garanticen su permanencia y el tránsito entre los grados y niveles educativos, en un proceso mediante el cual logren aprendizajes significativos y una formación integral.

Durante varios sexenios, desde la Secretaría de Educación Pública se ha reconocido que en el país existen problemas de desigualdad y exclusión social, por lo que se impulsaron acciones de atención enfocadas en diversos grupos socialmente vulnerables. A pesar de esto, es notorio que las políticas dirigidas a dichos grupos no han sido suficientes, tal y como se afirma en el Diagnóstico del Programa S244 Inclusión y Equidad Educativa (SEP, 2014). Uno de estos grupos es el constituido por niñas, niños y adolescentes (NNA) de familias de jornaleros agrícolas migrantes.

En México, la población infantil que muestra los mayores porcentajes de rezago educativo, repetición y abandono escolar son los menores de edad que, junto con sus familias, se desplazan de sus territorios de origen para incorporarse a las actividades productivas agrícolas en diversas regiones del país. Infantes que, no obstante el esfuerzo gubernamental realizado por más de tres décadas en la implementación de diversos programas educativos, permanecen sin tener pleno acceso a una educación con calidad y equidad (Rojas, 2014, p. 193).

Según la Red por los Derechos de la Infancia en México, los niños(as) de las familias jornaleras migrantes están marcados por la crueldad y la injusticia de la pobreza extrema, circunstancia que los convierte en un grupo de alta vulnerabilidad social (REDIM, 2018, p. 1). Dicho grupo también es considerado una de las subpoblaciones infantiles que en México no asiste a la escuela (Galeana, 2016).

Son muchas las aristas desde las cuales es posible estudiar la situación educativa de los NNA de familias jornaleras agrícolas migrantes. En este estudio, se reconoce la importancia que los docentes pueden tener en la tarea de crear oportunidades de aprendizaje dirigidas a los NNA que se inscriben en un sistema creado para ellos. Debido a esto, se considera necesario hacer una revisión de las prácticas pedagógicas desplegadas por los educadores de alumnos migrantes que se incorporan a la educación formal, específicamente las realizadas en centros escolares depen- 
dientes del Programa para la Inclusión y Equidad Educativa (PIEE) en el estado de Chihuahua. Se pretende que en dicha revisión participen directamente los propios docentes, pues son ellos quienes tienen la posibilidad real de problematizar y diseñar estrategias que mejoren los procesos educativos, al tener en cuenta contextos específicos y atender situaciones concretas y, con esto, beneficiar a la niñez migrante. Es necesario dejar claro que, si bien se considera de gran importancia el papel de los docentes en la creación de oportunidades de aprendizaje, esto no implica que se les responsabilice del éxito o fracaso escolar de NNA de familias migrantes.

En relación con la atención educativa que requieren las NNA de familias de jornaleros agrícolas migrantes, el Instituto Nacional para la Evaluación de la Educación (INEE) emitió cuatro amplias directrices cuyo propósito es vincular las decisiones que se tomen en el sistema educativo, con las necesidades de la niñez migrante ${ }^{1}$. Cobra especial importancia la atención a los procesos de formación docente en tales directrices. La segunda establece lo siguiente: asegurar la incorporación de un enfoque educativo intercultural, así como la disponibilidad, idoneidad y desarrollo profesional del personal con funciones de docencia. El propósito de esta norma es este: mejorar la enseńanza y el logro educativo de los alumnos mediante un desarrollo curricular pertinente y la gestión de procesos que garanticen la estabilidad laboral y la profesionalización de los docentes y de otras figuras educativas (INEE, 2016a, p. 28).

En el caso del estado de Chihuahua, la Secretaría de Educación y Deporte $(\mathrm{SEyD})$ reconoció las directrices y respondió al INEE con una serie de acciones, entre las cuales destacan la mejora del desempeńo docente a partir de la realización de programas de especialización en atención educativa a NNA de familias de jornaleros agrícolas migrantes, así como el fortalecimiento de los contenidos de interculturalidad en los programas de formación inicial de docente (INEE, 2016b, p. 1).

Asimismo, el Programa de Educación a la Niñez Migrante, dependiente del Departamento de Educación Indígena, Migrante y Menonita, ubicado en la Dirección de Atención a la Diversidad y Acciones Transversales, lleva a cabo una serie de estrategias para capacitar a educadores que se desempeńan en centros escolares de NNA de familias agrícolas migrantes.

Durante el ciclo agrícola 2017, se efectuaron periódicamente reuniones regionales de consejo técnico, que se constituyeron en espacios donde se enfatizó el análisis de temas académicos. La dinámica de dichas reuniones tuvo como núcleo la presentación de estrategias didácticas específicas, basadas en situaciones vivenciales y lúdicas. En el inicio del ciclo agrícola 2018, se planteó a los educadores de migrantes una propuesta para trabajar con el método de proyectos. Es necesario observar

${ }^{1}$ El INEE utiliza los resultados de las evaluaciones para elaborar directrices que fortalezcan la calidad y la equidad de los servicios educativos en México. Las directrices son propuestas de intervención pública, dirigidas a autoridades educativas responsables de ejercer el presupuesto y de aplicar estrategias de atención a las necesidades educativas específicas de la población. 
y dar seguimiento a este tipo de procesos formativos que se generan en la entidad, además de desarrollar, paralelamente, diseños de formación alterna.

\section{DIVERSAS OPCIONES EN LA FORMACIÓN DE DOCENTES}

En el ámbito educativo de hoy, se desarrollan propuestas de formación docente que conciben la práctica profesional como la aplicación de conocimientos cuya validez ha sido establecida previamente. Estas propuestas han recibido fuertes críticas, pues se considera que representan una «racionalización tecnológica de la enseñanza» que reduce la función del docente al cumplimiento de prescripciones externamente determinadas, "perdiendo de vista el conjunto y el control sobre su tarea» (Contreras, 1999, p. 21).

Es necesario explorar perspectivas de formación docente que partan de un horizonte epistemológico y sociopedagógico distinto al de los modelos concebidos desde la racionalización técnica; perspectivas que posibiliten a los propios enseñantes analizar y reflexionar sobre sus prácticas de enseñanza, de manera tal que este ejercicio de autoconocimiento profesional favorezca mayores niveles de autonomía en su desempeño. Este es, asimismo, uno de los factores que les permitirá reconocer las características de sus alumnos -los NNA de familias jornaleras migrantesy generar respuestas ante las problemáticas específicas que estos enfrentan.

Para impulsar ese proceso de autonomía docente, hasta el momento se ha identificado que es necesario lo siguiente:

- Reconocer que, en general, existe una brecha entre los lineamentos estandarizados o los supuestos bajo los cuales funciona el sistema educativo, y las condiciones específicas en las que se desarrolla la práctica pedagógica.

- Considerar que los docentes construyen conocimientos pedagógicos en el día a día de su práctica.

- Caracterizar la formación docente como un proceso que se lleva a cabo de manera constante, en ámbitos formales e informales.

- Establecer que es posible potencializar los procesos de formación, no esperar a que se presenten de manera natural o espontánea, por lo que es posible diseñar dispositivos específicos que propicien y fortalezcan su desarrollo.

Respecto a los supuestos del funcionamiento educativo, Terigi (2008) identifica cuatro principios: simultaneidad, descontextualización, presencialidad y cronosistema, los cuales pueden servir como referentes para analizar la praxis docente en contextos concretos (ver figura 1).

La disociación entre estos supuestos y las situaciones a las que se enfrentan los docentes es un fenómeno identificable en el ámbito educativo; sin embargo, es mayor en la educación que se ofrece a la población migrante, dadas sus características y las condiciones en que se da el proceso de enseñanza y aprendizaje. Una consecuencia inmediata de este fenómeno es que exige a los docentes generar de manera constante acciones diferenciadas para responder a los procesos cotidianos que se pre- 


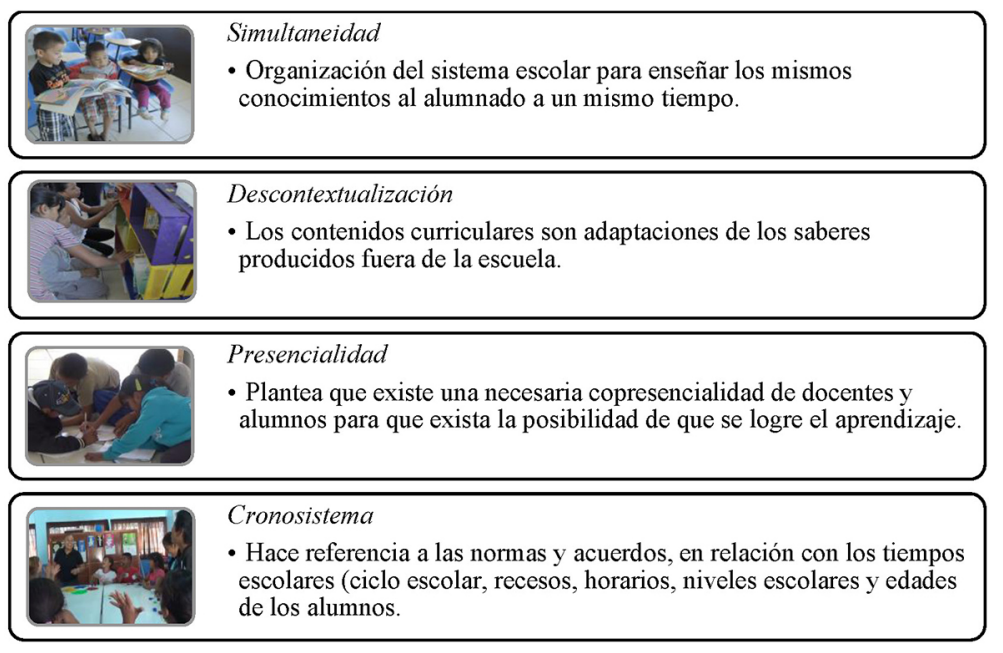

Fuente: elaboración propia, basada en Terigi (2008). Fotografías de Bruno García.

Figura 1. Supuestos del sistema educativo.

sentan en la escuela. Así, una condición previa al diseño de estrategias formativas dirigidas a estos profesores es conocer las principales características de sus prácticas, más allá de suponer que se ajustan a los supuestos del sistema educativo o aplican de manera uniforme los lineamientos pedagógicos establecidos por expertos.

Sobre el papel activo que los docentes asumen en la construcción de conocimientos pedagógicos, para Tardif (2004), los saberes presentes en las prácticas de los profesores tienen diferentes orígenes: profesionales, disciplinarios, curriculares y experienciales. Sin desconocer la importancia del resto de los saberes, resalta la de los conocimientos de tipo experiencial, a los que conceptualiza como saberes prácticos, cuyo origen es el trabajo diario de los profesores, quienes los desarrollan conforme a la cotidianeidad de su labor y con base en su conocimiento del entorno. De manera adicional, es preciso establecer que los saberes experienciales se producen en redes de interacciones en las cuales se encuentran inmersos los docentes. Dichas redes pueden presentarse tanto en contextos institucionales como no formales, con condiciones y características particulares que influyen en la construcción de dichos saberes (Tardif, 2004).

En referencia a los procesos de formación docente, Anijovich, Cappelletti, Mora y Sabelli (2009) también reconocen que no deben partir de prescripciones. Valoran las experiencias profesionales del enseñante y consideran que para establecer procesos de formación docente es necesario hacer una revisión inicial de dos conceptos básicos: trayecto formativo y dispositivo pedagógico, este último vinculado directamente a la reflexión.

Con respecto al trayecto formativo, para las citadas autoras, es de especial relevancia comprender la formación como un trayecto que se construye de manera individual, secuenciada y continua. Esta idea constituye un rechazo a los méto- 
dos de formación totalmente predefinidos, que no abonan a una formación integral. Sobre el concepto de dispositivo pedagógico, representa un plan para organizar el recorrido de la formación de los docentes, el cual posibilita «la valoración de la experiencia profesional a través de la biografía de un sujeto que [...] estructura sus formas de hacer y de pensar, y que pareciera tener conocimientos de los cuales no da cuenta, aunque los utiliza permanentemente en su accionar» (Anijovich et al., 2009, p. 22).

A partir de las nociones anteriores, resultan primordiales los posicionamientos formativos que centran su atención en la reflexión de los docentes acerca de sus prácticas, con el propósito de mejorarlas. Además, emerge como tema relevante la revisión de dispositivos y recursos pedagógicos que impulsen entre los docentes procesos de reflexión, individuales y colectivos.

Hay, en la literatura científica, diversos estudios que documentan el uso de dispositivos asociados a procesos de reflexión. Para los fines del presente trabajo, resultan de particular interés las investigaciones que tematizan sobre la utilización de videograbaciones como generadores de reflexión en los docentes. Fredericksen, Sipusic, Sherin y Wolfe (1998) refieren el trabajo con videoportafolios para documentar el ejercicio docente y efectuar un seguimiento de las acciones realizadas en la práctica pedagógica; Paquay y Wagner (2005) consideran que el video propicia una práctica docente reflexiva, al posibilitar procesos de autorreflexión y autorregulación; Perrenoud (2005) afirma que las experiencias de videoformación desarrolladas en Francia por Mottet son un ejemplo de cómo favorecer la toma de conciencia por parte del docente acerca de sus propias prácticas.

En cuanto al trabajo con docentes en formación, Tochon (2007) ha experimentado el uso del video como una herramienta que permite estimular reflexiones compartidas en grupos de estudio, a fin de establecer vínculos entre teoría y práctica, aspecto coincidente con Carbonneau y Hétu (2005), quienes establecen el trabajo con videos derivados de prácticas reales, asociados a estrategias de discusión colectiva de grupos conformados hasta por 35 integrantes.

En México, Sánchez y Ortega (2012) realizaron videoestudios en escuelas públicas urbanas, a partir de los cuales generaron conversaciones y reflexiones colectivas entre docentes, acerca de sus formas de enseñar. De manera similar, Sherin (2007) ha documentado experiencias en torno a la reflexión docente a partir de la organización de videoclubs, espacios donde los enseñantes reflexionan acerca de aspectos específicos de su propia práctica.

\section{ASPECTOS METODOLÓGICOS}

Esta investigación se basa en un enfoque metodológico cualitativo. La necesidad de conocer las prácticas pedagógicas que se despliegan en la educación proveída a población migrante, desde las voces de quienes enseñan a esa población, dictó la elección metodológica. Según Nagy (2017), desde una aproximación cualitativa, es preciso recurrir a métodos analíticos que favorezcan la comprensión subjetiva, con el fin de generar teorías explicativas de la realidad. Las metodolo- 
gías interpretativas privilegian la experiencia vivida y conservan el mundo de las narrativas de los participantes, en aras de abordar o acercarse al conocimiento de un tema o problema.

En esta primera etapa de la investigación, se aplicó un cuestionario y se efectuaron entrevistas semiestructuradas. El propósito del cuestionario fue recopilar información básica de los educadores: sexo, edad, idioma, nivel de escolaridad y experiencia docente con población migrante. La aplicación de entrevistas semiestructuradas buscó obtener una caracterización, desde la experiencia de los educadores, de aspectos básicos de su práctica docente y reflexiones en torno a esta: organización grupal, planeación, actividades, medios para la enseñanza, estrategias de evaluación. Las entrevistas se asociaron con la observación de fragmentos de videograbaciones de prácticas pedagógicas en las que habían participado los entrevistados; estas videograbaciones apoyaron los comentarios y respuestas por parte del docente entrevistado y la elaboración de preguntas por parte del investigador.

El trabajo de campo se realizó durante los meses de julio a octubre del ciclo agrícola 2017. En general, la estancia en las escuelas fue de tres días. El primer día se explicó a los profesores el propósito del trabajo a realizar y se aplicó un cuestionario, además de solicitar su permiso para videograbar una de sus clases. El segundo día se efectuó la videograbación de un fragmento de la jornada escolar; el tercer día se aplicó una entrevista, a partir de la observación de algunas escenas videograbadas, que se presentaron a los docentes.

Las 25 videograbaciones de clase obtenidas corresponden a los tres niveles de educación básica y tienen una duración promedio de 80 minutos. En todos los casos se revisó que el material videograbado contara con elementos para suscitar análisis referidos a la definición del propósito de la clase, actividades variadas, uso de medios para la enseñanza y acciones relacionadas con procesos de evaluación.

Las entrevistas acerca de la práctica docente tuvieron una duración promedio de 38 minutos. La duración de las entrevistas fue muy variable, de 13 a 75 minutos. Las diferencias dependieron principalmente de la disponibilidad de tiempo de los enseñantes, además del interés que mostraron por observar el video de su práctica y los comentarios que surgieron en torno a las actividades observadas o los desempeńos o producciones de los alumnos o de ellos mismos.

La estrategia de análisis de las entrevistas de los docentes fue de tipo inductivo, basada en los lineamientos de la teoría fundamentada (Corbin, 2010). Partiendo de las declaraciones de los educadores entrevistados, se identificó una serie de temas y se construyó una matriz comparativa, lo cual posibilitó la agrupación y contraste de las características adjudicadas a la práctica pedagógica.

\section{HALLAZGOS INICIALES}

Este apartado se conforma de dos secciones; de manera inicial, una descripción breve de las características de los docentes entrevistados; posteriormente, el registro o mostración de las definiciones que realizan en torno a sus prácticas docentes: 


\section{A) Perfil de los educadores entrevistados}

Las entrevistas se aplicaron a 25 educadores; 18 mujeres y 7 hombres. El promedio etario de este grupo es de 31 años: el de las mujeres, de 28 años, y el de los hombres, de 37. Casi la totalidad de los educadores son monolingües, hablan español. Dos de ellos (un hombre y una mujer) son bilingües; además de español, hablan su idioma materno, el rarámuri.

En lo relativo a su formación académica, los educadores entrevistados presentaron perfiles profesionales muy diversos. Algunos concluyeron sus estudios de educación media superior y actualmente realizan estudios en alguna licenciatura o ingeniería. Incluso se identificó el caso de una docente que cursa una maestría en educación. La mayoría de los educadores cuenta con estudios de licenciatura relacionados con la educación, aunque también se ubicaron educadores egresados de otros ámbitos. Algunos señalaron, además, haber cursado diplomados en diversos temas (neurolingüística, educación virtual, ciencias de la educación, mediación social, migración) o cursos y talleres (educación inicial, enfermería, computación, secretariado, topografía).

La experiencia docente que reportan los educadores resulta muy variada. Han laborado durante diferentes periodos en los niveles de educación básica, media superior y superior, tanto en instituciones públicas como privadas. Su práctica en educación básica ha sido como Instructores Comunitarios (IC) o, en periodos recientes, Líderes para la Educación Comunitaria (LEC), del Consejo Nacional para el Fomento Educativo (CONAFE). En dicha institución, algunos desarrollaron actividades como instructores, capacitadores y/o asistentes educativos. Asimismo, mencionaron haber trabajado como docentes de primarias regulares, tanto públicas como privadas. En el nivel de secundaria, algunos indicaron haberse desempeñado como profesores de diferentes asignaturas, en secundarias públicas. Otros más relataron haber trabajado en preparatorias particulares, o en un Centro de Bachillerato Tecnológico Agropecuario. Y hay también, entre ellos, quienes han laborado en educación superior, en instituciones privadas, a nivel licenciatura.

Acerca de su experiencia laboral en centros escolares para población migrante, algunos educadores iniciaron con la introducción del Programa de Educación Básica para Niños y Niñas de Familias Jornaleras Agrícolas Migrantes (PRONIM) en el estado de Chihuahua, en el año 2010, es decir, cuentan con siete años de experiencia. En contraparte, se identifican casos de enseñantes que se incorporaron al trabajo docente con migrantes durante los últimos meses del ciclo agrícola 2017.

En general, refirieron que no han recibido capacitación específica para trabajar con NNA migrantes. Algunos consideraron que durante su formación profesional (Licenciatura en Intervención Educativa y Licenciatura en Preescolar y Primaria para el Medio Indígena) revisaron características generales de grupos en situación de vulnerabilidad, sin hacer una revisión específica de poblaciones migrantes.

Considerando lo anterior, este grupo de educadores ha tenido que enfrentarse y responder al reto que representa la docencia con NNA de familias jornaleras agrícolas migrantes. Lo han hecho a partir de sugerencias de docentes más experimentados, asesorías cortas, como las realizadas en reuniones de consejo técnico, al 
trasponer experiencias de docencia adquiridas en otros contextos o derivadas de los procesos en los que han participado como alumnos, pero sobre todo mediante la experiencia directa. Esta situación viene a confirmar que las instituciones no forman con un perfil específico que resulte eficaz y adecuado a la población migrante (Rojas, 2011). Reitera, además, la importancia del saber experiencial descrito por Tardif (2004) y la necesidad de atender trayectos formativos específicos (Anijovich et al., 2009).

\section{B) Reflexiones de LOS DOCENTES EN TORNO A SU PRÁCTICA PEDAGÓGICA}

Las clases que se videograbaron fueron determinadas por los propios educadores; ellos seleccionaron la temática y la forma de organizar el grupo. Al observarlas, plantearon descripciones y reflexiones en torno a su trabajo.

Según lo expuesto por los educadores, la organización grupal es un aspecto que se identifica como nodal, debido a su impacto en el resto de los factores que se presentan en sus prácticas. En la tabla 2, es posible advertir la diversidad de formas de organización grupal que se presentaron en las clases videograbadas.

\begin{tabular}{|c|c|c|c|c|c|c|}
\hline \multicolumn{7}{|c|}{$\begin{array}{l}\text { TABLA 2. NIVELES Y TIPO DE ORGANIZACIÓN } \\
\text { DE LOS GRUPOS VIDEOGRABADOS EN CLASE }\end{array}$} \\
\hline \multirow[t]{2}{*}{ Preescolar } & $P_{R}$ & ARIA & SECUNDARIA & & Multinivei & \\
\hline & unigrado & multigrado & multigrado & $\begin{array}{l}\text { preescolar/ } \\
\text { primaria }\end{array}$ & $\begin{array}{l}\text { primaria/ } \\
\text { secundaria }\end{array}$ & $\begin{array}{c}\text { preescolar/ } \\
\text { primaria/ } \\
\text { secundaria }\end{array}$ \\
\hline 5 casos & 6 casos & 5 casos & 1 caso & 5 casos & 1 caso & 2 casos \\
\hline
\end{tabular}

Fuente: elaboración propia.

Sobre esta diversidad en la organización grupal, los educadores estimaron que al interior de las aulas la situación se presenta de manera más compleja, debido a las características del alumnado: niveles de adquisición del lenguaje escrito, situaciones de extraedad y la presencia de alumnos que inician el aprendizaje del español como segunda lengua. Ante esto, los enseñantes realizan una serie de ajustes en sus prácticas. Ajustan los aspectos siguientes:

\section{La organización áulica:}

- En algunos casos, los educadores determinan la asignación de alumnos en los diferentes grados escolares, en consonancia con su edad. Según explicaron, ubicar a alumnos con extraedad (que están en un proceso inicial de adquisición de la lectoescritura) en grados superiores los alienta a asistir a la escuela, pues les evita sentir vergüenza por trabajar con los niños más pequeños. Esta decisión ha llevado a los educadores a plantear actividades diferenciadas y atención individual para los alumnos con extraedad. 
- Asimismo, recurren a la formación de grupos de alumnos según el manejo que tienen de la lectoescritura (sin considerar grados escolares), lo que permite presentar de manera simultánea dos o tres actividades con diferente nivel de complejidad para los grupos así organizados.

- Otra estrategia que aplican es la realización de actividades de lectoescritura para todo el grupo, en las cuales los alumnos participan según sus posibilidades. En este tipo de actividades, valoran como una ventaja el apoyo que se presenta entre los alumnos para su realización.

\section{El plano curricular:}

- La mayoría de los educadores definieron, de una u otra manera, que el propósito de las escuelas para la niñez migrante es enseñarles a leer y a escribir, además de las operaciones matemáticas básicas. A partir de esto, los docentes toman decisiones como las que se describen a continuación:

- Dedicación exclusiva a la aplicación de actividades relacionadas con lectura y escritura y, en menor medida, operaciones matemáticas básicas, con la consecuente eliminación de otros contenidos programáticos.

- Definición de un listado de contenidos que los educadores consideran importante que los niños migrantes deben aprender. Algunas formas de selección de contenidos observadas son arbitrarias, basadas en el sentido común. En otros casos, se retoman contenidos de libros de texto oficiales o de editoriales privadas y, excepcionalmente, de los programas de estudio.

- Realización de actividades tomadas de diversos medios: libros, ficheros y, en muchos casos, de páginas de internet.

Los procesos de evaluación y el tránsito escolar:

- Casi la totalidad de los educadores hizo referencia a procesos de monitorización constante durante sus clases. Esto les permite identificar problemáticas específicas que presentan los alumnos y darles apoyo concreto.

- Los educadores relataron que efectúan una valoración de los aprendizajes que los alumnos presentan, con base en observación directa de las actividades que llevan a cabo durante las clases. En algunos casos, se realizan seguimientos a partir de la elaboración de portafolios o carpetas e, incluso, se llegan a aplicar pruebas objetivas.

- En referencia a la acreditación de grados escolares, comentaron que puede realizarse en lapsos breves, conforme a criterios que ellos mismos establecen y relacionados con el desempeño de los alumnos, principalmente en la lectura y la escritura. 


\section{ALGUNAS CONCLUSIONES DE ESTA ETAPA DE INVESTIGACIÓN}

La aplicación de entrevistas asociadas a videograbaciones facilitó las respuestas de los educadores entrevistados. Además, propició el planteamiento de preguntas referidas a aspectos concretos de la práctica videograbada. La revisión global de las declaraciones de los educadores permite aseverar que en sus respuestas aluden a situaciones específicas de su práctica docente. Esto constituyó una ventaja, pues centraron su atención en elementos de las actividades realizadas por ellos y sus alumnos, evitando así definir su quehacer docente desde aspectos normativos.

Las declaraciones de los docentes, en general, se ubican en un nivel descriptivo. Desde la explicativa de Hatton y Smith (como se citó en Anijovich et al., 2009), sus relatos se ubican en una modalidad reflexiva de menor profundidad: descripciones no reflexivas, que se limitan a informar acerca de los hechos; reflexiones descriptivas, que establecen razones o justificaciones basadas en opiniones o experiencias propias.

Desde ahora, resulta indispensable subrayar las ventajas de trabajar con videograbaciones para favorecer procesos iniciales de reflexión en los docentes. Sin embargo, es necesario diseñar y explorar dispositivos capaces de suscitar reflexiones dialogadas, en las que los profesores reconozcan y valoren sus conocimientos previos, a la vez que nutren esa reflexión con resultados de investigaciones y teorías pedagógicas, de modo que logren construir concepciones de mayor profundidad, así como la capacidad crítica suficiente para sopesar el entorno en que se produce la enseñanza y el aprendizaje. Esta profundización de la actividad reflexiva implica no solo una auténtica concienciación de sus propias necesidades y saberes, sino además la posibilidad real de sustentar nuevas prácticas pedagógicas; en última instancia, significa enriquecer el trabajo de educar. Sobre tal aspecto, Edelstein plantea la siguiente consideración:

El saber docente no se conforma solo desde la práctica; se nutre también en las teorías que dotan a los sujetos de variados puntos de vista y perspectivas de análisis que les permiten una acción contextualizada sobre la base de la comprensión de los contextos históricos, sociales, culturales, organizacionales en los que se desenvuelven profesionalmente (Edelstein, 2012, p. 67).

\section{Prospectos de LA INVESTigación}

En una siguiente etapa indagadora, se analizarán trabajos que tematizan sobre los cambios en los niveles de reflexión de los docentes como factor de profesionalización; específicamente, se revisará la indagación de Loubet y Jacobo (2013), cuyo propósito fue conocer la reflexión social que llevan a cabo educadores de migrantes; asimismo, el estudio exploratorio de Loubet (2018), que se planteó como objetivo identificar la construcción de una reflexión social y crítica sobre la función social del fenómeno educativo, a partir de una estrategia de intervención basada en un semi- 
nario de sociología de la educación, cuyos participantes eran, a la vez que alumnos, docentes. Ambos proyectos de investigación nutren las tesis de la docencia reflexiva.

Además, se analizará la experiencia derivada de la investigación de Ávila, Hernández, Echeverría y Zambrano (2016), la cual tiene como uno de sus objetivos promover la reflexión de los docentes a partir de sesiones de videoanálisis, para generar propuestas de innovación.

Igualmente, un ejercicio que es necesario emprender empíricamente en la próxima etapa de esta investigación es la revisión -entre pares o grupos de educadores de población migrante- de videograbaciones de prácticas docentes, que permita reconocer el tipo y niveles de reflexión colectiva que llevan a cabo.

Recibido: 02-04-2019. Aceptado: 24-05-2019 


\section{REFERENCIAS}

Anijovich, R., Cappelletti, G., Mora, S. y Sabelli, M.J. (2009). Transitar la formación pedagógica. Dispositivos y estrategias. Argentina: Paidós.

Ávila, L.A., Hernández, A.K., Echeverrís, M.R. y Zambrano, A. (2016). «El acompañamiento a docentes de educación básica para nińos jornaleros migrantes y desarrollo docente». Revista Innovación Educativa, 16 (71), 111-132.

Carbonneau, M. y Hétu, J.C. (2005). «La formación práctica de los maestros y el nacimiento de una inteligencia profesional», en La formación profesional del maestro. Estrategias y competencias. México: Fondo de Cultura Económica.

Contreras, J. (1999). La autonomía del profesorado. Espańa: Morata.

Corbin, J. (2010). «La investigación en la teoría fundamentada como medio para generar conocimiento profesional», en La teoría fundamentada: una metodología cualitativa. México: Universidad Autónoma de Aguascalientes.

Edelstein, G. (2011). Formar y formarse en la enseñanza. Argentina: Paidós.

Frederiksen, J., Sipusic, M., Sherin, M. y Wolfe, E. (1998). «Video Portfolio Assessment: Creating a framework for viewing the functions of teaching», disponible en http://www.tandfonline.com/doi/abs/10.1207/s15326977ea0504_1.

Galeana, R. (2016). Identificación de subpoblaciones de niños que no asisten a la escuela y caracterización de los factores asociados a la problemática. Conformando un marco inicial para la construcción de indicadores. México: INEE.

INEE (2016a). Directrices para mejorar la atención educativa de niñas, niños y adolescentes de familias de jornaleros agrícolas migrantes. México: Autor.

INEE (2016b). «Respuestas a las directrices. Chihuahua», disponible en http://www.inee.edu.mx/ index.php/respuestas-directrices-migrantes-2016.

Loubet, R. (2018). «Contribución de la sociología en la formación docente, un ejercicio de intervención en México». Revista de la Asociación de Sociología de la Educación, 11 (1), 5-20.

Loubet, R. у Јасово, H.M. (2013). "La reflexión sociofuncional y crítica de los profesores de programas de educación para la población infantil migrante». Revista Internacional de Educación para la Justicia Social. 2 (1), 195-212.

NAGY, Sh. (2017). The practice of qualitative research. United States of America: Sage.

PaQuaY, L. y Wagner, M.C. (2005). «Formación continua y videoformación: qué habilidades se deben priorizar», en La formación profesional del maestro. Estrategias y competencias. México: Fondo de Cultura Económica.

Perrenoud, P. (2005). «El trabajo sobre el habitus. Análisis de las prácticas y toma de conciencia», en La formación profesional del maestro. Estrategias y competencias. México: Fondo de Cultura Económica.

Red POR los derechos de la infancia en México (2018). «Callejera. En Otras Infancias». México: Autor, disponible en http://www.derechosinfancia.org.mx/index.php?contenido=pagina\&id=14\&id_opcion=55.

Rojas, T.J. (2014). Exclusión y politica social para la atención de los jornaleros agrícolas migrantes. México: Universidad Pedagógica Nacional. 
RoJas, T.J. (2011). Inequidades. La educación primaria de niñas y niños jornaleros agrícolas migrantes. México: Universidad Pedagógica Nacional.

SÁnchez, J.S. y Ortega, M.C. (2012). Videoestudios de formación docente en la escuela. Cómo reflexionan los maestros sobre sus prácticas de enseñanza. México: Universidad Pedagógica Nacional.

SEP (2014). «Diagnóstico del Programa S244. Inclusión y Equidad Educativa». México: Autor, disponible en http://www.sep.gob.mx/work/models/sep1/Resource/5009/1/images/diagnostico_del_programa_s244.pdf.

Sherin, M. (2007). «The development of teachers' professional vision in Video Clubs», en Video research in learning sciences. United States of America: Lawrence Erlbaum.

TARDif, M. (2004). Los saberes del docente y su desarrollo profesional. España: Narcea.

Terigi, F. (2008). «Organización de la enseñanza en los plurigrados de las escuelas rurales». Tesis de Maestría presentada en la Facultad Latinoamericana de Ciencias Sociales, Sede académica Argentina, disponible en http://repositorio.flacsoandes.edu.ec/bitstream/10469/1266/1/ TFLACSO-2009FZT.pdf.

Tochon, F. (2007). «From video cases to video pedagogy: a framework for video feedback and reflection in pedagogical research praxis", en Video research in learning sciences. United States of America: Lawrence Erlbaum. 
